\title{
Labyrinthe
}

\section{Colloque « L'Italie à la fin du Moyen Âge : les caractères originaux dans le cadre européen »}

"L'Italia alla fine del Medioevo : i caratteri originali nel quadro europeo ", Fondazione Centro studi sulla civiltà del tardo Medioevo, VIII Convegno Internazionale di Studi. San Miniato, 28 septembre- $1^{\mathrm{er}}$ octobre 2000.

Pierre Savy

\section{(2) OpenEdition}

\section{Journals}

Édition électronique

URL : http://journals.openedition.org/labyrinthe/869

DOI : $10.4000 /$ labyrinthe.869

ISSN : 1950-6031

Éditeur

Hermann

Édition imprimée

Date de publication : 1 janvier 2001

Pagination : 119-122

Référence électronique

Pierre Savy, "Colloque «L'Italie à la fin du Moyen Âge : les caractères originaux dans le cadre européen » », Labyrinthe [En ligne], 8 | 2001, mis en ligne le 25 mai 2005, consulté le 09 mai 2019. URL http://journals.openedition.org/labyrinthe/869 ; DOI : 10.4000/labyrinthe.869

Ce document a été généré automatiquement le 9 mai 2019.

Propriété intellectuelle 


\section{Colloque « L'Italie à la fin du Moyen Âge : les caractères originaux dans le cadre européen »}

«L'Italia alla fine del Medioevo : i caratteri originali nel quadro europeo ", Fondazione Centro studi sulla civiltà del tardo Medioevo, VIII Convegno Internazionale di Studi. San Miniato, 28 septembre- ${ }^{\mathrm{er}}$ octobre 2000.

\section{Pierre Savy}

1 Le thème retenu cette année pour le colloque organisé par la «Fondazione Centro studi sulla civiltà del tardo Medioevo ", qui consacre ses travaux à la civilisation du bas Moyen Âge, en particulier à l'espace italien, avait a priori de quoi surprendre : les " caractères originaux » de l'Italie "dans le cadre européen ", voilà une expression dont le premier terme peut bien faire écho au grand livre de Marc Bloch ${ }^{1}$ et le second à une Europe à la construction de laquelle l'Italie a depuis toujours été associée (de cette Europe contemporaine, il était du reste question dans la présentation du colloque); mais voilà une expression un peu floue. Le texte accompagnant le programme du colloque apportait quelques explications : il s'agissait de présenter à grands traits l'Italie du bas Moyen Âge dans une optique comparatiste, de la situer dans une perspective européenne, d'insister sur les spécificités majeures de l'espace italien autour du $\mathrm{XV}^{\mathrm{e}}$ siècle. La démarche, très générale, ou synthétique, - ou macro-analytique -, excluait toute monographie. Le cadre d'analyse restait large et seules étaient prises en compte les questions importantes, sans prétention à la systématicité, ni à l'exhaustivité : le but n'était pas de couvrir, par addition des différentes interventions, tout le territoire italien ${ }^{2}$.

De plus, il devait être question, explicitement, des aspects structurels par opposition à la culture et aux mentalités, qui doivent bientôt faire l'objet d'un autre colloque : après l'infrastructure, la superstructure, en somme ${ }^{3}$. Était donc explicitement adopté un cadre conceptuel fort classique, qui faisait de ce colloque une expression de " macro-histoire ", braudélienne dirait un Français, pratiquant un « étagement » des réalités à la manière des 
Annales $^{4}$. Quoi qu'on en pense, ce cadre conceptuel avait le mérite de la clarté, et celui surtout de ne pas exclure des interventions fort diverses: ce thème et cette approche larges devaient permettre, comme l'affirma Giorgio Chittolini dans son introduction, de faire le point sur la question, si débattue en Italie, des différents niveaux d'analyse (micro et macro), et d'offrir une vision de la fin du Moyen Âge italien à la fois globale et à jour, ce qui n'est pas fréquent. Le colloque entendait en somme dire l'essentiel le mieux possible, non pas exposer des idées nouvelles sur des personnages méconnus ou des questions rarement abordées ${ }^{5}$.

Cinq thèmes successifs formaient l'ossature de ces journées : le milieu et les paysages ; la société; l'Église et la société; les institutions politiques; et l'économie. Parmi les participants, les Italiens, milanais notamment, étaient bien sûr nombreux ; Allemands et Anglais étaient bien représentés ; il n'y avait pas de Français parmi les intervenants ${ }^{6}$.

Disons maintenant un mot des interventions, souvent de grande qualité, qu'il nous fut donné d'entendre. Aldo Schiavone commença en dressant le bilan de la présence, massive, de l'antiquité romaine dans le Moyen Âge italien, et salua la réhabilitation de la notion de tardoantico, sur l'utilité de laquelle il insista dans sa présentation nettement problématique et historiographique. L'intervention de Francesca Bocchi sur "les caractères originaux des villes italiennes" traita de problèmes plus strictement urbanistiques, comme la situation excentrée des cathédrales italiennes faute de place dans le tissu urbain pré-chrétien. La société fut abordée de diverses manières, un peu croisées : Claudio Donati, évoquant « les noblesses italiennes », insista sur l'originalité des nobles italiens parmi les nobles européens. En Italie, en effet, la question si débattue de la continuité et des origines franques n'a pas cours; la noblesse italienne est intimement liée à la ville, mais est aussi très diverse d'un lieu à l'autre. Certaines interventions furent limpides, notamment celle d'Arnold Esch, qui présenta en parallèle, avec clarté et audace, les sociétés urbaines italienne et allemande. L'histoire politique fut abordée notamment par Francesco Somaini - qui offrit une vue d'ensemble de la papauté au $\mathrm{xv}^{\mathrm{e}}$ siècle -, Alison Brown, qui rendit compte de la crise du républicanisme florentin, et Guido Castelnuovo, qui parla des officiers et des « appareils de gouvernement » en Italie. Giorgio Chittolini, président du comité scientifique de la fondation, professeur à l'Università degli Studi de Milan, offrit une bonne synthèse, osant l'approche comparatiste, sur les principautés en Italie et en Europe, insistant sur l'origine souvent communale et urbaine des principautés italiennes et sur la situation particulière du Nord de l'Italie, en raison de la place qu'y occupe l'empereur et de l'absence de grande monarchie. Une des dernières interventions, stimulante et contestable, fut celle de Mario Sbriccoli sur les deux types de justice, «négociée » et " hégémonique », et l'imposition de celle-ci aux dépens de celle-là au cours du Moyen Âge. L'intervention de Lorenzo Epstein, générale elle aussi ( "L'économie italienne et l'économie européenne »), fut, comme les autres travaux de cet historien économiste, particulièrement remarquable, insistant sur le rôle des crises (la Peste, creative destruction selon l'expression de Schumpeter, et la crise de la fin du Moyen Âge, qui modifie la hiérarchie économique européenne) et montrant la fonction dominante de la ville dans l'économie du territoire. Evelyn Welch mit en avant les spécificités de la consommation italienne par rapport aux consommations flamandes du $\mathrm{xV}^{\mathrm{e}}$ siècle.

5 On peut, bien sûr, regretter quelques absences : la diplomatie, dont l'étude nous paraît utile pour pratiquer une histoire comparée (pour situer l'Italie dans le cadre européen), ne fut abordée que par Riccardo Fubini, à propos de Florence; l'histoire militaire stricte 
ne profita pas du renouvellement récent qu'a connu cette branche ancienne de l'histoire ${ }^{7}$; un autre thème, peut-être en raison de son énormité et de l'âpreté des débats qu'il a occasionnés, fut négligé : l'État. Néanmoins, ces défauts ne ternissent pas le tableau positif d'un colloque qui a su, par exemple, ne pas délaisser l'économie, ce qui est assez rare pour être notable.

Quels sont donc, pour conclure, les caractères originaux de l'Italie de la fin du Moyen Âge ? Marino Berengo, historien moderniste italien, né en 1928, est mort en août 2000 ; il a profondément marqué l'historiographie italienne de la deuxième moitié du $\mathrm{XX}^{\mathrm{e}}$ siècle, et son grand livre, L'Europe des villes, a été publié peu avant sa disparition ${ }^{8}$. On parla beaucoup de lui, notamment au début du colloque, qui fut pour ainsi dire placé sous son " patronage » : rien d'étonnant par conséquent à ce que la ville y occupât une telle place. La ville, caractère original de l'Italie ? Rien là de faux mais rien là de nouveau, si bien que l'envie venait parfois de rappeler, comme l'ont fait certains historiens anglais, que les milieux féodaux, la dimension rurale et agraire, l'agriculture, les campagnes continuèrent d'occuper une place centrale dans l'économie, la société et la civilisation italiennes9.

La ville fut bien, à ce colloque de San Miniato, considérée comme «le principe idéal des histoires italiennes ${ }^{10} »$. La publication des actes constituera un volume utile dans un paysage historiographique où ce type de parcours demeure rare; de plus, par-delà la diversité des propos et des sujets traités, elle constituera une nouvelle affirmation, assez consensuelle, du primat du fait urbain dans l'Italie du bas Moyen Âge, et par là, un hommage à un maître disparu.

\section{NOTES}

1. Les Caractères originaux de l'histoire rurale française, Oslo, Leipzig, Paris, Londres et Cambridge, 1931.

2. Le morcellement de l'histoire et donc de l'historiographie italiennes est tel que souvent, c'est une addition de monographies régionales qui fait le plan des histoires générales (c'est le cas, pour l'essentiel, de la Storia d'Italia dirigée par Giuseppe Galasso, publiée à Turin depuis 1979 et qui, au demeurant, fait référence).

3. On lisait dans la présentation du colloque qu'il devait s'agir « d'aspects plus structurels : politique, société, économie, église ", tandis qu'un deuxième colloque prévu en 2002 traiterait « des thèmes et des aspects d'histoire de la culture et de la mentalité ». (« Un primo convegno previsto dal 28 settembre al $1^{\circ}$ ottobre darà conto di aspetti più strutturali : politica, società, economia, chiesa ; un secondo convegno previsto per il 2002 affronterà aspetti e temi di storia della cultura e della mentalità. »)

4. On note cependant qu'à la différence de l'étagement classique des Annales des années 1950 et 1960, et dans une optique propre à l'historiographie italienne, l'Église - comme institution - et les institutions politiques furent, à ce colloque, considérées comme des aspects structurels, si bien que leur traitement ne fut pas différé au deuxième colloque. 5. L'intervention de Kaspar Elm sur deux réformateurs du $\mathrm{xv}^{\mathrm{e}}$ siècle, personnages peu connus, fait exception. 
6. En l'absence de Philippe Contamine, de l'Université Paris IV, dont je ne sais si l'intervention sera publiée dans les actes du colloque, l'historien italien Guido Castelnuovo, qui enseigne à Chambéry, fut le seul représentant de l'Université française. 7. Sur ce renouvellement, dans une bibliographie abondante, la publication du colloque de Lucques de mai 1998 (Condottieri e uomini d'arme nell'Italia del Rinascimento (1350-1450), dir. Giorgio Chittolini et Bruno Figliuolo) permettra de faire le point.

8. L'Europa delle città. Il volto della storia urbana europea tra Medioevo ed Età moderna, Turin, 1999. Berengo est aussi l'auteur d'un livre important sur les nobles et les marchands de Lucques au XVI ${ }^{\mathrm{e}}$ siècle (Nobili e mercanti nella Lucca del Cinquecento, Turin, 1965).

9. Philip James Jones, « Economia e società nell'Italia medievale : la leggenda della borghesia ", dans Ruggiero Romano et Corrado Vivanti (dir.), Dal feudalesimo al capitalismo, Storia d'Italia, Annali, 1, Turin, 1978, p. 185-372 ; du même, The Italian city-State. From Commune to Signoria. 500-1300, Oxford et New York, 1997 ; et aussi Trevor Dean, Land and Power in Late medieval Ferrara. The Rule of the Este, 1350-1450, Cambridge, 1988. Les campagnes ne furent pas, certes, passées sous silence : Giovanni Cherubini dressa un tableau clair et général des « sociétés rurales », Anna Maria Rapetti évoqua les «paysages ruraux ».

10. Pour reprendre le célèbre titre de Carlo Cattaneo, « La città considerata come principio ideale delle istorie italiane », 1858, Notizie sulla Lombardia. La città, éd. Giuseppe Armani, Milan, 1979, p. 115-169. 